\title{
Transformative Bildung im naturwissenschaftlichen Unterricht
}

Hochschulprof. Dr. Thorsten Kosler, Pädagogische Hochschule Tirol

Im Diskurs um Bildung für nachhaltige Entwicklung wurde immer wieder die Forderung erhoben, Lernende müssten in die Lage versetzt werden, unser Denken in grundlegender Weise zu hinterfragen und zu verändern (Sterling, 2001; 2003; Scott \& Gough 2003; Vare \& Scott 2007; Wals 2010; Wals \& Dillon 2013). Entsprechend wird im Positionspapier zur Umsetzung von SDG $4^{1}$ in Österreich des Fachbeirates „Transformative Bildung/ Global Citizenship Education“ der Österreichischen UNESCO-Kommission (2019) das Ziel 4 der Agenda 2030 als Auftrag für eine transformative Bildung gesehen (ebd., S. 2). Mit transformativer Bildung ist demnach eine Bildung gemeint, die zu einer notwendigen sozial-ökologischen gesellschaftlichen Transformation beiträgt (ebd., S. 14). Eine solche Bildung erfordere eine eigene Pädagogik, die im Anschluss an Singer-Bodrowski (2016a, 2016b) anhand von drei Merkmalen charakterisiert werden könne: Transformative Bildung zielt auf den Wandel von individuellen „Bedeutungsperspektiven“, versteht sich als kollektiver Bewusstwerdungs- und Emanzipationsprozess und fragt auch nach der notwendigen Veränderung von Kulturen und Strukturen (des Bildungswesens), die emanzipatorisches Lernen behindern (ebd., S. 14). Unklar bleibt an dieser

1 Mit ,SDG 4“ ist das Sustainable Development Goal 4 („Inklusive, gleichberechtigte und hochwertige Bildung gewährleisten und Möglichkeiten lebenslangen Lernens für alle fördern") aus der Agenda 2030 (vgl. dazu Rieckmann in diesem Band) gemeint. 
Stelle, wie der Wandel individueller Bedeutungsperspektiven als individueller Lernprozess mit der Transformation kollektiver Denkstrukturen im Kontext einer nachhaltigen Entwicklung zusammenhängt. Aus meiner Sicht liegt hier ein Theoriedefizit in der Debatte um Bildung für nachhaltige Entwicklung vor. Solange unklar ist, inwiefern individuelle Lernprozesse $\mathrm{zu}$ einer gesellschaftlichen Transformation im Sinne einer nachhaltigen Entwicklung beitragen können, bleibt auch unklar, wie und was die einzelnen Unterrichtsfächer zu eben dieser Transformation beitragen können.

Um diesen Zusammenhang näher zu beleuchten, werden im Kapitel 1 drei Theorieansätze transformativer Bildung daraufhin analysiert, welchen Ertrag sie für die Klärung dieses Zusammenhanges bieten. Als Erstes wird der Ansatz Sterlings im Kontext seiner sustainability education analysiert, da Sterling innerhalb der internationalen Debatte um ,Education for Sustainable Development‘ der Einzige ist, der sich dieser Frage detailliert gewidmet hat. In der Regel wird dieser Frage gar nicht im Detail nachgegangen, sondern es wird dazu auf den in der angelsächsischen Erwachsenenbildung verbreiteten Ansatz des transformativen Lernens verwiesen (vgl. Wals \& Dillon, 2013). Daher wird auch dieser Ansatz dargestellt und analysiert. Da beide Ansätze aus meiner Sicht den Zusammenhang zwischen individuellem Lernen und gesellschaftlicher Transformation nur unbefriedigend klären können, wird als drittes der Ansatz transformatorischer Bildung aus der deutschsprachigen Bildungstheorie dargestellt und gezeigt, inwiefern dieser Ansatz weiter führt. In Kapitel 2 wird dann auf dieser Basis beispielhaft eine Möglichkeit erläutert, wie individuelles Lernen im naturwissenschaftlichen Unterricht so gestaltet werden kann, dass es als Beitrag zu einer gesellschaftlichen Transformation im Kontext einer nachhaltigen Entwicklung gesehen werden kann.

\section{Konzeptionen transformativer Bildung}

Die Idee, Bildung müsse zu einer gesellschaftlichen Transformation beitragen, schließt letztlich direkt an eines der Gründungsdokumente der Debatte um

Kapelari, Suzanne (Hg.), Vierte „Tagung der Fachdidaktik“ 2019:

„Interdisziplinäre fachdidaktische Diskurse zur Bildung für nachhaltige Entwicklung“

(C) 2020 innsbruck university press, ISBN 978-3-99106-019-2, DOI 10.15203/99106-019-2 
Bildung für nachhaltige Entwicklung, dem Artikel 36 der Agenda 21, an. Dort wurde die Idee formuliert, dass hinausgehend über Bindestrich-Pädagogiken eine Neuausrichtung des gesamten Bildungssystems nicht mehr an wirtschaftlichem Wachstum, sondern an nachhaltiger Entwicklung notwendig sei (Hopkins, 2012, S. 23ff.). In die drei im Folgenden dargestellten Konzeptionen transformativer Bildung sind jeweils eine Vielzahl von theoretischen Ansätzen eingeflossen, mit denen die jeweiligen Autoren versucht haben, Details ihrer Konzeptionen auszuarbeiten. Zwei Theorieansätze finden sich dabei in allen drei Konzeptionen und bieten bereits eine erste Möglichkeit, den Zusammenhang von individuellem Lernen und der Transformation kollektiver Denkstrukturen zu beleuchten. Das ist zum einen die Idee des Paradigmenwechsels von Kuhn (1976) und zum anderen die Lerntheorie von Bateson (1987). Im Anschluss an diese Ansätze von Kuhn und Bateson (1.1) wird zunächst das transformative Lernen nach Sterling (1.2), das transformative Lernen aus der angelsächsischen Erwachsenenbildung (1.3) und der transformatorische Bildungsbegriff der deutschsprachigen Bildungstheorie (1.4) vorgestellt und im Hinblick auf den Zusammenhang zwischen individuellem Lernen und kollektivem Umdenken analysiert.

\subsection{Kuhns Paradigmenwechsel und Batesons Lerntheorie}

Kuhn (1976) unterscheidet in seinem Modell von der Struktur wissenschaftlicher Revolutionen als Paradigmenwechsel zwei Phasen des Wandels in der Naturwissenschaft. In Phasen der Normalwissenschaft besteht der Wandel darin, neue Gesetze zu entdecken, die das bestehende Wissensgebäude um einen weiteren Baustein ergänzen, ohne dass es dabei zu Veränderungen von Grundbegriffen oder der Art und Weise kommt, wie die Welt betrachtet wird. In Phasen wissenschaftlicher Revolution werden dagegen Entdeckungen gemacht, die mit den traditionellen Begriffen nicht in Einklang zu bringen seien. Kuhn gibt in einer Zusammenfassung seines Modells (Kuhn, 1981, S. 32f.) drei Charakteristika an, die revolutionäre Veränderungen in seinem Sinne miteinander gemein haben: Erstens haben sie eine systemische

Kapelari, Suzanne (Hg.), Vierte „Tagung der Fachdidaktik“ 2019:

„Interdisziplinäre fachdidaktische Diskurse zur Bildung für nachhaltige Entwicklung“

(C) 2020 innsbruck university press, ISBN 978-3-99106-019-2, DOI 10.15203/99106-019-2 
Struktur, insofern sie nicht etappenweise, Schritt für Schritt, vollzogen werden können, da eine Anzahl untereinander verbundener Gesetzmäßigkeiten revidiert wird. Zweitens vollziehe sich dabei ein Bedeutungswandel, insofern die „Art und Weise, wie Worte und Sätze mit den Naturphänomenen verbunden sind“ (ebd., 34), sich verändert. Und drittens vollzieht sich nach Kuhn ein „grundlegender Wandel des Modells, der Metapher oder der Analogie“ (ebd., S. 36), der für die Mitglieder einer Forschungsgemeinschaft leitend ist (vgl. Kosler, 2016, S. 17-20). Kuhns Arbeit hat in der Wissenschaftstheorie eine umfangreiche Kontroverse ausgelöst und auch die Rezeptionsgeschichte in der Naturwissenschaftsdidaktik ist komplex (Matthews, 2004). Aber die Grundidee, die viele ErziehungswissenschaftlerInnen inspiriert hat, ist wohl die folgende: Wenn ausgerechnet in den vermeintlich, exakten' Naturwissenschaften ein revolutionärer Umbruch im Denken einer Forschungsgemeinschaft möglich ist, dann könnte dies bedeuten, dass ein solch plötzlicher Umbruch des Denkens auch im lernenden Individuum möglich ist. Letztlich setzt Kuhns Theorie des kollektiven Umdenkens sogar voraus, dass ein solch plötzliches Umdenken in Individuen möglich ist, da das kollektive Umdenken ja gerade darin besteht, dass in kurzer Zeit ein relevanter Anteil an Mitgliedern einer Forschungsgemeinschaft ein solches Umdenken individuell vollzieht. In der Naturwissenschaftsdidaktik war Kuhns Ansatz die Inspirationsquelle für die Idee des Conceptual Change, also eines ebenso plötzlichen Bedeutungswandels, der sich im Denken von Lernenden vollzieht. Dazu haben Posner, Strike, Hewson und Gertzog (1982) einige der Kriterien, die Kuhn dafür angibt, unter welchen Bedingungen sich ein solcher Umbruch in Forschungsgemeinschaften vollzieht, auf die Situation von lernenden Individuen übertragen. Auf die Frage, wie ein neues Paradigma inhaltlich zustande kommt, sagt Kuhn nichts. Er beschreibt lediglich Bedingungen, die in Forschungsgemeinschaften erfüllt sein müssen, damit sich ein neues Paradigma in einer Forschungsgemeinschaft gegenüber dem bestehenden durchsetzen kann. Damit bleibt auch unklar, wie ein individueller Lernprozess zu einem kollektiven Umdenken beitragen kann.

Bateson (1987) fasst seine Stufen des Lernens zunächst recht abstrakt. Lernen betrachtet er als Veränderung einer Reaktion auf einen Stimulus. Er geht davon

Kapelari, Suzanne (Hg.), Vierte „Tagung der Fachdidaktik“ 2019:

„Interdisziplinäre fachdidaktische Diskurse zur Bildung für nachhaltige Entwicklung“

(C) 2020 innsbruck university press, ISBN 978-3-99106-019-2, DOI 10.15203/99106-019-2 
aus, dass Organismen den Strom an Erfahrungen und Handlungen im Leben in Abschnitte unterteilen, sie miteinander vergleichen und sie als gleichartig oder verschiedenartig klassifizieren. Solche Abschnitte fasst Bateson als Kontexte auf. Zudem unterscheidet Bateson zwei Ordnungen von Fehlern: Ein Organismus kann Informationen korrekt nutzen, die ihm verraten, aus welcher Menge von Handlungsalternativen er wählen muss, dann aber die falsche Alternative innerhalb dieser Menge wählen. Oder der Fehler liegt schon in der Wahl der falschen Menge von Handlungsalternativen.

Lernen I definiert er dann als Änderung der Reaktion innerhalb derselben Menge an Handlungsalternativen.

Lernen II fasst er als Änderung im Prozess des Lernens I und definiert es als Änderung der Wahl der Menge der Handlungsalternativen, aus denen gewählt werden kann.

Lernen III fasst er als Änderung im Prozess des Lernens II und definiert es als korrigierende Veränderung im System von Handlungsalternativen, aus denen gewählt werden kann.

Lernen IV fasst er schließlich als Änderung im Lernen III und gesteht zu, dass dieses wohl nicht bei erwachsenen, lebenden Organismen auf der Erde vorkommt.

Um Batesons Lernstufenmodell zu verstehen, ist es zentral, wie die Mengen von Handlungsalternativen zustande kommen. Eine Möglichkeit, sich dies zu veranschaulichen, besteht darin, diese Mengen von Handlungsalternativen im Sinne der Sprechakttheorie von Austin (1976) und Searle (1969) zu deuten (vgl. Kosler 2016, S. 67). So weist Austin darauf hin, dass mit einem Satz wie ,Ich komme morgen wieder' unterschiedliche Handlungen vollzogen werden können. Er kann als Drohung, Versprechen oder Prognose gemeint sein. Entsprechend kann ein Gesprächspartner den Satz als Handlung richtig oder falsch verstehen. Die richtige Interpretation der Handlungsintention liefert dem Interpreten dann im Sinne Batesons eine sinnvolle Menge von Handlungsalternativen. In Batesons Modell lässt sich verstehen, wie individuelle Lernprozesse mit kollektiven Veränderungsprozessen zusammenhängen. Die Handlungskontexte, die nach Bateson jeweils mit einer Menge an sinnvollen Handlungsalternativen verbunden sind, sind keine rein 
individuellen Angelegenheiten. Dass sich eine Handlung als Drohung, als Versprechen oder auch als Prognose deuten lässt, ist ja gerade eine kollektive Vereinbarung. Zu der Frage, wie es zu neuen kollektiv ausgehandelten Mengen von Handlungsalternativen kommen kann, findet sich bei Bateson allerdings keine Antwort.

\subsection{Transformatives Lernen im Rahmen einer Sustainability Education}

Im Diskurs um Bildung für nachhaltige Entwicklung ist es insbesondere Sterling (2001, 2003, 2008, 2011, 2014) gewesen, der im Rahmen seines Konzeptes einer Sustainability Education die Idee transformativen Lernens in den Mittelpunkt gestellt hat. Dabei geht Sterling davon aus, dass sich aktuell zwei Weltbilder gegenüber stehen: Ein mechanistisches Weltbild der Moderne, das er als mechanistisch, positivistisch und dualistisch (Sterling, 2001, S. 50) charakterisiert und ein entstehendes ökologisches Weltbild im Kontext der Postmoderne (ebd., S. 49f.). Zur Erläuterung verweist er auf AutorInnen, die ausführen, die Moderne sei an einer Maschinen-Metapher orientiert, während das entstehende ökologische Weltbild an der Metapher des Organismus oder des lebenden Systems orientiert sei. Er wirft die Frage auf, inwieweit die bestehenden unterschiedlichen pädagogischen Ansätze der Erziehungswissenschaft nicht letztlich einem mechanistischen Weltbild zugeordnet werden müssen. Seinen Ansatz einer Sustainability Education ordnet er dem entstehenden ökologischen Weltbild zu. Im Mittelpunkt steht dabei die Ausbildung eines ganzheitlichen systemischen Denkens (,whole systems thinking“, ebd., S. 51), das unser Weltbild hin zu einem ökologischen Weltbild und unser pädagogisches Denken sowie unsere pädagogische Praxis verändern soll (ebd., S. 51). Um die individuelle Seite des Lernens näher zu beleuchten, unterscheidet Sterling (ebd., S. 15f.) drei Ordnungen des Lernens: Lernen erster Ordnung findet danach innerhalb akzeptierter Grenzen statt und ist adaptiv, insofern es die zugrundeliegenden Werte unhinterfragt und unverändert lässt. Lernen zweiter Ordnung sei ein kritisches, reflektiertes Lernen, das die Annahmen, die das Lernen erster Ordnung beeinflussen,

Kapelari, Suzanne (Hg.), Vierte „Tagung der Fachdidaktik“ 2019:

„Interdisziplinäre fachdidaktische Diskurse zur Bildung für nachhaltige Entwicklung“

(C) 2020 innsbruck university press, ISBN 978-3-99106-019-2, DOI 10.15203/99106-019-2 
untersucht. Lernen dritter Ordnung schließlich ermögliche es uns, Dinge anders zu sehen. Es sei kreativ, beinhaltet ein tiefes Bewusstsein von alternativen Weltbildern und Wegen, Dinge zu tun. Ein solches Lernen dritter Ordnung versteht Sterling als transformatives Lernen. Die radikale Bewegung hin zu Nachhaltigkeit erfordere individuell und gesellschaftlich ein solches transformatives Lernen.

Um die individuellen und kollektiven Transformationsprozesse genauer $\mathrm{zu}$ fassen, zieht Sterling (2003) eine Vielzahl von Theorieentwürfen heran. Von zentraler Bedeutung ist für ihn dabei der Begriff des Paradigmas, da sein Ansatz der Sustainability Education einerseits darauf zielt, zum gesellschaftlichen Wandel vom mechanistischen hin zu einem ökologischen Weltbild beizutragen, und er seinen pädagogischen Ansatz selbst als Versuch deutet, ein ökologisches Paradigma in der Pädagogik zu entwickeln, das er als Subparadigma des ökologischen Weltbildes versteht. Weltbilder werden bei ihm dabei mit Paradigmen weitestgehend gleichgesetzt (ebd., S. 120). Dazu weitet er Kuhns Modell des Paradigmenwechsels in Forschungsgemeinschaften sowohl auf Individuen als auch auf Gesellschaften aus und kombiniert es mit Batesons Stufenmodell des Lernens, um sein eigenes Modell der Ordnungen des Lernens genauer zu fassen. Das Hinterfragen eines bestehenden Paradigmas deutet er im Sinne Batesons als Lernen II: „In sum, where the whole cultural paradigm is at stage, my understanding is that Learning II is a realization of the limits of a dominant paradigm, and a move towards an alternative, even where the basis for that alternative may not be fully understood" (ebd., S. 136f.). Lernen III deutet er dann entsprechend als Beherrschung der Klasse der Paradigmen. Wer Lernen III vollzogen hat, ist danach in der Lage, zwischen verschiedenen Paradigmen zu wechseln.

Sterling (ebd.) nimmt sich auch eines Problems an, das er in seiner früheren Arbeit (2001, S. 50) nur beiläufig erwähnt. Er sieht in der Postmoderne zwar ein alternatives Weltbild, aufgrund des ihm zufolge darin enthaltenen Relativismus sei dieses aber nicht als ökologisches Weltbild geeignet. Es könne daher nur einen Zwischenschritt auf dem Weg zu einem entstehenden ökologischen 
Weltbild darstellen, das er als revisionäre Postmoderne beschreibt (Sterling 2003, S. 147f.). Dabei würde insbesondere der Dekonstruktivismus den Raum für ein ökologisches Weltbild öffnen, zugleich aber dessen Möglichkeit bestreiten. Dazu bezieht er sich insbesondere auf Lyotard (1986), der ausführt, große Erzählungen hätten in gegenwärtigen Gesellschaften einen Glaubwürdigkeitsverlust erlitten. Solche großen Erzählungen würden zum einen dazu neigen, Sprachspiele zu vereinheitlichen und die tatsächliche Vielfalt bestehender Sprachspiele auszublenden. Zudem würden sie dazu neigen, deskriptive und normative Aussagen zu vermischen (vgl. dazu Koller 2012, S. 88). Sterling unternimmt zwar nicht den Versuch eine ökologische Erzählung zu konstruieren, wie dies andere (z.B. O’Sullivan 1999) versucht haben. Aber er gibt einige Aspekte an, in denen sich ein ökologisches Weltbild vom bestehenden unterscheiden müsste (Sterling 2001, S. 170f.). Darunter eine Veränderung ,of conception of an essentially dead and inert world, to an animate, dynamic and ultimately sacred world" (ebd.). Dabei deutet sich an, dass sich in einem solchen Weltbild wieder Beschreibung und Bewertung vermischen. Angesichts des Einwandes von Lyotard bleibt damit unklar, wie im Modell von Sterling ein Übergang von einem postmodernen $\mathrm{zu}$ einem ökologischen Weltbild möglich ist.

\subsection{Transformatives Lernen in der angelsächsischen Erwachsenenbildung}

Mit der Formulierung, transformative Bildung würde auf einen Wandel individueller Bedeutungsperspektiven zielen, verweist der Fachbeirat ,Transformative Bildung/Global Citizenship Education“ auf die Diskussion um transformatives Lernen in der angelsächsischen Erwachsenenbildung. Dieser Ansatz geht auf Mezirow zurück, der ursprünglich Lernprozesse in Wiedereinstiegsprogrammen für Frauen an community-colleges, die nach einer längeren Unterbrechung ans College oder an den Arbeitsmarkt zurückkehren, untersucht hat (Mezirow 1978a, 1978b; Baumgartner 2012). Nach Mezirow erlangen die Frauen in diesen Kursen im günstigsten Fall ein kritisches Bewusstsein von den kulturellen und psychologischen Annahmen, die die

Kapelari, Suzanne (Hg.), Vierte „Tagung der Fachdidaktik“ 2019:

„Interdisziplinäre fachdidaktische Diskurse zur Bildung für nachhaltige Entwicklung“

(C) 2020 innsbruck university press, ISBN 978-3-99106-019-2, DOI 10.15203/99106-019-2 
Weise, wie wir uns selbst und unsere Beziehungen zu anderen sehen, und die Weise, wie wir unser Leben strukturieren, beeinflussen (Mezirow 1978b, S. 101). Genau dies bezeichnet er als Lernen über Bedeutungsperspektiven (ebd.). Er führt explizit in Analogie zu Kuhns Theorie vom Wechsel wissenschaftlicher Paradigmen aus: „A meaning perspective refers to the structure of cultural assumptions within which new experience is assimilated to - and transformed by - one's past experience. It is a personal paradigm for understanding ourselves and our relationships“ (ebd.). Als mögliche Auslöser für solche Lernprozesse identifiziert er Herausforderungen und Dilemmata des Erwachsenenlebens. Das Ziel eines solchen Prozesses ist für Mezirow 1978 noch ein kohärentes, autonomes Subjekt. Der Prozess wird im Wesentlichen als Rollenwechsel verstanden (ebd.; vgl. Kosler, 2016, S. 71ff.). Zur weiteren Ausgestaltung seines Ansatzes zieht Mezirow ab 1981 dann die Unterscheidung von drei Erkenntnisinteressen, die Habermas (1968) in seiner Frankfurter Antrittsvorlesung getroffen hat, heran (Mezirow 1981, S. 3ff.; vgl. Kosler, 2016, S. 73f.). Den Prozess der Perspektiventransformation deutet er nun in Anlehnung an Habermas als „emancipatoric process of becoming critical aware of how and why the structure of psycho-cultural assumptions come to constrain the way we see ourselves and our relationships, reconstituting this structure to permit a more inclusive and discriminating integration of experience and acting upon these new understandings" (Mezirow, 1981, S. 6). Später beschreibt Mezirow das Werk von Habermas als soziolinguistischen Rahmen seiner Transformationstheorie (Mezirow, 1997, S. 143). Bedeutungsperspektiven sind bei Mezirow nun wiederum aus Bedeutungsschemata zusammengesetzt: „Ein Bedeutungsschema stellt ein bestimmtes Wissen, bestimmte Überzeugungen, Werturteile und Empfindungen dar, die in einer Interpretation ihren Ausdruck finden. Bedeutungsschemata sind die konkreten Manifestationen unserer gewohnheitsmäßigen Orientierung und Erwartungen (Bedeutungsperspektiven) und übertragen diese allgemeinen in besondere Erwartungen, die uns bei unseren Handlungen leiten“ (ebd., S. 36). Als Beispiel gibt er den Ethnozentrismus als Mittelpunkt einer Bedeutungsperspektive an und bestimmt dann rassische und sexuelle Klischees als Bedeutungsschemata innerhalb dieser Perspektive. Auf Basis der oben erläuterten Lerntheorie von Bateson, die er als

Kapelari, Suzanne (Hg.), Vierte „Tagung der Fachdidaktik“ 2019:

„Interdisziplinäre fachdidaktische Diskurse zur Bildung für nachhaltige Entwicklung“

(C) 2020 innsbruck university press, ISBN 978-3-99106-019-2, DOI 10.15203/99106-019-2 
„bahnbrechende Analyse“ charakterisiert (ebd., S. 77), beschreibt er vier Formen des Lernens in der Erwachsenenbildung. Er reformuliert dazu Batesons Stufenmodell mit Hilfe seiner Begriffe des Bedeutungsschemas und der Bedeutungsperspektive. Lernen II ist danach ein Lernen, das Bedeutungsschemata verändert, während Lernen III die Veränderung der Bedeutungsperspektive beinhaltet. Damit unterscheidet Mezirow die folgenden vier Formen des Lernens (ebd., S. 77f.):

1. Lernen durch Bedeutungsschemata, als Differenzierung und Verfeinerung der als gegeben angesehenen Bedeutungsschemata oder durch Lernen innerhalb der Struktur eines Bezugsrahmens.

2. Erlernen neuer Bedeutungsschemata, als Schaffung neuer Bedeutungen, die mit bestehenden Bedeutungsperspektiven vereinbar sind, insofern diese dadurch in ihrem Anwendungsbereich erweitert werden.

3. Lernen durch Transformation von Bedeutungsschemata, die als dysfunktional erkannt wurden.

4. Lernen durch Transformation ganzer Bedeutungsperspektiven.

Damit hat Mezirow ein differenziertes Raster vorgelegt, in dem verschiedene Formen des Lernens unterschieden werden können. Durch die Adaption der Lerntheorie von Bateson liefert Mezirows Ansatz eine analoge Antwort auf die Frage, wie individuelles Lernen mit kollektiven Bewusstwerdungs- und Emanzipationsprozessen zusammenhängt. Die Bedeutungsperspektiven bei Mezirow sind ebenfalls keine rein individuelle Angelegenheit. Bedeutungsperspektiven sind auch bei ihm kollektiv entwickelte Möglichkeiten, unseren Strom an Eindrücken und Erfahrungen zu strukturieren und mit Sinn zu versehen. Offen bleibt bei Mezirow allerdings, wie individuelle und kollektive Veränderungsprozesse ineinandergreifen, um neue Bedeutungsperspektiven hervorzubringen. Genau dies wäre im Hinblick auf das Verhältnis individueller und gesellschaftlicher Transformationsprozesse im Kontext einer nachhaltigen Entwicklung relevant. Dass diese Frage bei Mezirow nicht auftritt, liegt vermutlich daran, dass sein Ansatz daran orientiert ist, wie individuelle Transformationsprozesse im Kontext der feministischen Bewegung unterstützt werden können. Dabei scheint mir eine theoretische Neubestimmung der Rollenverteilung von Frauen und Männern in heterosexuellen Paarbeziehungen

Kapelari, Suzanne (Hg.), Vierte „Tagung der Fachdidaktik“ 2019:

„Interdisziplinäre fachdidaktische Diskurse zur Bildung für nachhaltige Entwicklung“

(C) 2020 innsbruck university press, ISBN 978-3-99106-019-2, DOI 10.15203/99106-019-2 
bereits Teil des Rahmens gewesen zu sein, der durch die Politisierung dieser Thematik durch den Feminismus im Bewusstsein großer Teile der Bevölkerung angekommen war. Das Ziel der von Mezirow beschriebenen Seminare bestand dann darin, Menschen, die für sich bereits beschlossen hatten, aufgrund solcher Einsichten ihr Leben zu ändern, dabei zu unterstützen. Vermutlich sind manche Menschen auch erst in solchen Seminaren $\mathrm{zu}$ entsprechenden Einsichten gekommen. Aber die eigentliche Aufgabe bestand darin, aufgrund solcher Einsichten, das eigene Fühlen und Handeln, insbesondere eingeübte Verhaltensroutinen, zu ändern. Dabei sind neue Handlungsweisen relativ einfach aus der theoretischen Analyse abzuleiten gewesen, z.B. eben nicht mehr jeden Abend dem Ehemann das Essen zuzubereiten. Die Ausgangslage scheint mir im Kontext einer nachhaltigen Entwicklung eine andere zu sein. Neue Bedeutungsperspektiven müssen ja erst entwickelt werden und es stellt sich die Frage, wie transformatives Lernen dazu beitragen kann.

\subsection{Der transformatorische Bildungbegriff der deutschsprachigen Bildungstheorie}

Aus meiner Sicht wäre der in der deutschsprachigen Bildungstheorie insbesondere von Kokemohr (1989, 1992, 2007), Marotzki (1990) und Koller (2012) entwickelte Begriff transformatorischer Bildung als Ansatzpunkt besser geeignet, um im Kontext einer nachhaltigen Entwicklung genauer zu fassen, wie individuelle und gesellschaftliche Transformationsprozesse aufeinander bezogen sind. Ausgangspunkt dieser Konzeption ist zunächst eine Unterscheidung von Lernen und Bildung, derzufolge Lernen als Prozess der Aufnahme, Aneignung und Verarbeitung neuer Informationen zu verstehen ist, während Bildung als Lernprozess höherer Ordnung $\mathrm{zu}$ verstehen ist, bei dem nicht nur neue Informationen angeeignet werden, sondern auch der Modus der Informationsverarbeitung sich grundlegend ändert (Koller, 2012, S. 15). Diese Unterscheidung wurde von Matrotzki (1990) und Kokemohr (1992) auch explizit als bildungstheoretischer Kern des Batesonschen Stufenmodells ausgewiesen. Die Grundidee dieses Ansatzes besteht in dem Vorschlag, Bildung

Kapelari, Suzanne (Hg.), Vierte „Tagung der Fachdidaktik“ 2019:

„Interdisziplinäre fachdidaktische Diskurse zur Bildung für nachhaltige Entwicklung“

(C) 2020 innsbruck university press, ISBN 978-3-99106-019-2, DOI 10.15203/99106-019-2 
als „Prozess der Be- oder Verarbeitung solcher Erfahrungen zu untersuchen, die der Subsumption unter Figuren eines gegebenen Welt- und Selbstentwurfs widerstehen“ (Kokemohr, 2007, 21). Kokemohr sieht es dann als gerechtfertigt an, von Bildung zu sprechen, „wenn der Prozess der Be- oder Verarbeitung subsumptionsresistenter Erfahrungen eine Veränderung von Grund legenden Figuren meines je gegebenen Welt- und Selbstentwurfs einschließt“ (ebd.). Koller hebt zwei Elemente besonders hervor, die aus seiner Sicht neu an Kokemohrs Vorschlag gegenüber Humboldts Bildungsbegriff sind. Zum einen bestimmt Kokemohr den Anlass für einen solchen Bildungsprozess als Krisenerfahrung, die in der Konfrontation mit einer Problemlage besteht, für deren Bewältigung sich das bisherige Welt- und Selbstverhältnis als nicht ausreichend erweist (Koller, 2012, S. 16). Zum anderen hebt er den Anspruch Kokemohrs hervor, seinen Bildungsbegriff als anschlussfähig für qualitativempirische Untersuchungen zu fassen (ebd., S. 16f.). Bildung mit Kokemohr und Koller als Transformation von Figuren des Welt- und Selbstverhältnisses von Menschen angesichts neuer Problemlagen, für deren Bewältigung die Figuren ihres bisherigen Welt- und Selbstverhältnisses nicht mehr ausreichen, zu fassen, kann dabei nicht als Definition, sondern eher als Vorschlag für die Form einer Definition verstanden werden. So benennt Koller vier Dimensionen, in denen eine nähere Bestimmung nötig ist (ebd., S. 17), die sich als Fragen formulieren lassen:

1. Wie lassen sich die Strukturen der Selbst- und Weltverhältnisse beschreiben?

2. Welche Problemlagen oder Krisenerfahrungen können transformatorische Bildungsprozesse auslösen? Und welche tun dies typischerweise?

3. Wie verlaufen solche Prozesse und welche Prozessstrukturen lassen sich dabei identifizieren? Wie kann Neues in einem solchen Prozess entstehen?

4. Auf welche Weise ist ein empirischer Zugang $\mathrm{zu}$ transformatorischen Bildungsprozessen möglich?

Im Kontext nachhaltiger Entwicklung stellt sich darüber hinaus die Frage, wie ein individueller Bildungsprozess als Beitrag $\mathrm{zu}$ einer gesellschaftlichen Transformation verstanden werden kann. Diese Frage stellt Koller selbst zwar nicht, aber es lassen sich mit ihm zumindest Ansatzpunkte für eine Antwort 
finden. So schließt Koller (ebd., S. 88ff.) an Lyotards „Der Widerstreit“ (1989) an, in dem dieser sich mit der Legitimation gesellschaftlicher Ordnung befasst. In der Interpretation Kollers, der ich hier folge, geht Lyotard nicht davon aus, dass Subjekte vor oder unabhängig von der Äußerung von Sätzen bestehen, sondern davon, dass sie in Sätzen als Sender, Empfänger oder Referenten situiert werden. Obwohl jeder Satz als eigenes Universum betrachtet wird, lassen sich Darstellungsarten von Universen und damit Satzfamilien unterscheiden, die verknüpft sind mit Regeln der Satzbildung. Entsprechend lassen sich beispielsweise deskriptive, präskriptive, evaluative, interrogative oder ostensive Sätze unterscheiden. Entscheidend ist nun, dass „Sätze unterschiedlicher Regelsysteme [...] nicht ineinander übersetzt werden“ (Lyotard, 1989, S. 92) können. Sätze können allerdings verkettet werden und solche Verkettungen konstituieren Diskursarten, die Lyotard als Regelsysteme höherer Ordnung betrachtet, die die möglichen Verkettungen unter einen bestimmten Zweck stellt. Als Beispiele zitiert Koller bei Lyotard unter anderem „Wissen, Lehren, Rechthaben, Verführen, Rechtfertigen, Bewerten, Erschüttern, Kontrollieren“ (Koller, 2012, S. 91). Solche Zwecke dürfen dabei nicht als Intentionen von Subjekten, sondern im Sinne von Strategien, die Spielern „auferlegt" (ebd.) werden, verstanden werden. Entsprechend ließen sich schließlich verschiedene Diskursarten wie wissenschaftliche, ökonomische, philosophische und narrative unterscheiden. Der besondere Clou besteht nun darin, dass es beim „Aufeinandertreffen von unterschiedlichen Diskursarten unvermeidlich zu einem Widerstreit (différend) kommt, d.h. zu einem Konflikt, der prinzipiell nicht zu schlichten ist, weil eine übergreifende Urteilsregel fehlt" (ebd., S. 92). Genau hierin besteht die Idee einer radikalen Heterogenität der Diskursarten.

Mit einer solchen Konzeption kann nun Koller im Hinblick auf die ersten beiden der erwähnten vier näheren Bestimmungen des Bildungsbegriffes zumindest Ansatzpunkte beschreiben. Zum einen lässt sich mit Lyotard verstehen, dass die Struktur von Welt- und Selbstverhältnissen als Resultat der Verkettung von Sätzen nach diskursiven Regeln $\mathrm{zu}$ fassen sei und die Positionierung von Subjekten im Verhältnis zur Welt und zu sich selbst damit 
nicht als Ausdruck einer der Sprache vorgängigen Subjektivität zu verstehen ist. Als Problemlagen, die Bildungsprozesse auslösen können, sind so Fälle von Widerstreit zu betrachten. Koller kann aus dem Rekurs auf Lyotard auch eine Minimalethik seiner Bildungstheorie ableiten: Diese besteht in der Anerkennung der radikalen Heterogenität der Diskursarten, aus der er die Forderung ableitet, ein bereits artikulierter Widerstreit müsse offen gehalten werden, um zu verhindern, dass durch Heranziehen einer vermeintlich übergeordneten Regel eine Seite der Möglichkeit, sich zu artikulieren, beraubt würde. Außerdem müsse auch dort, wo kein offener Konflikt zu erkennen sei, „,sondern ein Schweigen oder ein ,Gefühl' anzeigt, dass in der vorherrschenden Diskursart ,etwas“ nicht gesagt werden kann“ (ebd., 94), mit der Möglichkeit des Widerstreits gerechnet werden. Damit versteht Koller Bildung ,als jenen Prozess der Entstehung neuer Sätze und Diskursarten [...], der zur Anerkennung und zum Offenhalten des Widerstreits erforderlich ist“" (ebd., S. 97). So ist klar, dass durch die individuelle Artikulation neuer Sätze und die neuartige Verkettung von Sätzen neue Diskursarten geschaffen werden können.

Im Hinblick auf die Frage, wie individuelles Lernen und die Transformation kollektiver Denkstrukturen zusammenhängen, liefert Koller mit seiner Interpretation Lyotards eine völlig neue Perspektive. Subjekte denkt Lyotard gar nicht als unabhängig von Sätzen und Diskursarten. Subjekte situieren sich erst in der Äußerung von Sätzen und diese sind durch kollektiv ausgehandelte Regelsysteme, die Diskursarten, reguliert. Wenn ein individueller Bildungsprozess über die Artikulation neuer Sätze neue Diskursarten hervorbringt, dann ist Bildung bei Koller so immer ein Akt der Veränderung von Gesellschaft. Was bei Lyotard fehlt, sind allerdings Hinweise darauf, wie die Hervorbringung neuer Diskursarten $\mathrm{zu}$ bewerkstelligen ist. Lyotards Analyse weist bloß darauf hin, dass dies möglich sei.

Für die Beantwortung der Frage, wie im individuellen Bildungsprozess Neues entstehen kann, diskutiert Koller sieben verschiedene Theorieansätze. Darunter auch Kuhns Paradigmenwechsel in der Naturwissenschaft, da Kuhn selbst von einem plötzlichen Umschwung in seinem Denken über die aristotelische Physik

Kapelari, Suzanne (Hg.), Vierte „Tagung der Fachdidaktik“ 2019:

„Interdisziplinäre fachdidaktische Diskurse zur Bildung für nachhaltige Entwicklung“

(C) 2020 innsbruck university press, ISBN 978-3-99106-019-2, DOI 10.15203/99106-019-2 
ausgegangen war und dann nach dem Vorbild dieses individuellen Prozesses seine Theorie eines kollektiven Paradigmenwechsels formuliert hatte. Im Hinblick auf den Zusammenhang von individuellem Lernen und der Transformation kollektiver Denkstrukturen ist dabei insbesondere der von Koller herangezogene Ansatz von Derrida relevant. Dazu schließt Koller an Derridas Aufsatz „Signatur Ereignis Kontext“ (1988) an. Ich fasse dazu Kollers Interpretation zusammen. Während im gewöhnlichen Verständnis Kommunikation als Übermittlung eines Sinns aufgefasst wird (Derrida, 1988, S. 291; Koller, 2012, S. 127), zeigt Derrida, dass dies nur möglich ist, wenn der jeweilige Kontext begrenzt werden kann. Derridas These besteht nun darin, dass ein solcher Kontext nie völlig bestimmt und eingegrenzt werden kann. Dabei geht Derrida von einer bestimmten Form der Kommunikation, nämlich der Schrift aus. Nach dem gängigen Verständnis fassen wir die Schrift als ein Mittel zur Übermittlung von Sinn auf, welches es uns beispielsweise erlaubt, mit Personen zu kommunizieren, die sich nicht zur selben Zeit am selben Ort wie wir befinden. Derrida hebt nun hervor, dass die Schrift vom Empfänger und vom Sender abgelöst ist und dass die sie ausmachenden Schriftzeichen iterierbar, also wiederholbar, sind. Außerdem ist sie von den Intentionen und vom Bewusstsein des Senders losgelöst und damit dessen Kontrolle entzogen. Die Schrift ist abgelöst vom „Horizont des Sinns“ (Derrida, 1988, S. 299; Koller, 2012, S. 128), den Koller als Vorstellung eines geschlossenen und eindeutig feststellbaren Sinns interpretiert, und vom Kontext, wie sich am Beispiel des Zitats sehen lässt, in dem ein neuer Kontext aufgepfropft wird. Entsprechendes gilt für gesprochene Sprache, da auch sie nur funktionieren kann, wenn sie unabhängig von demjenigen, der etwas geäußert hat, und vom Kontext, in dem dies geschah, iterierbar ist. Diese Ablösbarkeit könne man beispielsweise an widersinnigen Äußerungen wie „der Kreis ist viereckig“ oder „das grün ist oder“" sehen. Und schließlich erläutert Koller, dass Derrida die Möglichkeit des Misslingens performativer Sprechakte, anders als Austin, nicht als Anomalie deutet. Ein Beispiel wäre die feierliche Äußerung eines Schauspielers im Rahmen eines Theaterstückes „Ich taufe dieses Schiff auf den Namen Anna“, mit dem das dem Schauspieler gegenüberstehende Objekt eben nicht getauft wird. Derrida vertritt im Gegenteil die Auffassung, das Gelingen

Kapelari, Suzanne (Hg.), Vierte „Tagung der Fachdidaktik“ 2019:

„Interdisziplinäre fachdidaktische Diskurse zur Bildung für nachhaltige Entwicklung“

(C) 2020 innsbruck university press, ISBN 978-3-99106-019-2, DOI 10.15203/99106-019-2 
performativer Sprechakte sei gerade an die Ausnahme gebunden, dass die Intention des sprechenden Subjektes anwesend ist. Daraus folgert Derrida, dass Kommunikation nicht als Übermittlung von Sinn verstanden werden dürfe und auch nicht Gegenstand eines hermeneutischen Verstehens sein könne, das auf die „Entzifferung eines Sinns oder einer Wahrheit“ (Derrida, 1988, S. 299; Koller, 2012, S. 129) ausgerichtet ist. Alternativ schlägt Derrida dann eine Lektüre vor, die der Zerstreuung und Vervielfältigung des Sinns folgt. Damit sei Derridas Konzept einer dekonstruktiven Lektüre zumindest angedeutet. Neue Lesarten würden durch den „Nachvollzug der potentiellen und nie völlig beherrschbaren Vieldeutigkeit eines Textes bzw. - übertragen auf Bildungsprozesse - einer Erfahrung“ (Koller, 2012, S. 129) entstehen. Koller betont dabei, dass damit, wie schon im hermeneutischen Ansatz, die Entstehung des Neuen als Prozess unablässiger Auseinandersetzung mit dem Gegenstand verstanden wird und insofern Derrida am Vorrang der Sache und der Notwendigkeit einer Infragestellung der eigenen Position der Leserin bzw. des Lesers festhalte (ebd.).

\subsection{Bildungstheoretische Schlussfolgerungen für den naturwissenschaft lichen Unterricht}

Kollers transformatorischer Bildungsbegriff eignet sich, um einen Zusammenhang zwischen einem individuellen Bildungsprozess und einem gesellschaftlichen Transformationsprozess herzustellen. Mit Lyotard ist ein transformatorischer Bildungsprozess als Hervorbringung neuer Sätze und Diskursarten zu verstehen, die einen Widerstreit zu bestehenden Diskursarten und Sätzen erzeugt oder einen solchen aufrecht erhält. Mit Derrida ist die dekonstruktive Lektüre eines Textes, die Koller auch auf Erfahrungen erweitert, eine Möglichkeit, um aus der nach Derrida bestehenden potentiellen Vieldeutigkeit neuen Sinn hervorzubringen. Für naturwissenschaftliches Denken bedeutet dies, dass aus einer dekonstruktiven Lektüre der Naturwissenschaft ein neuer Diskurs erzeugt werden kann, der im Widerstreit zum naturwissenschaftlichen Diskurs steht. Eine solche Dekonstruktion der

Kapelari, Suzanne (Hg.), Vierte „Tagung der Fachdidaktik“ 2019:

„Interdisziplinäre fachdidaktische Diskurse zur Bildung für nachhaltige Entwicklung“

(C) 2020 innsbruck university press, ISBN 978-3-99106-019-2, DOI 10.15203/99106-019-2 
Naturwissenschaft sehe ich dabei zunächst nicht als sinnvolles Ziel innerhalb naturwissenschaftlichen Unterrichts. Eine Dekonstruktion der Naturwissenschaft ist ein Weg, um einen widerstreitenden Diskurs über die Natur hervorzubringen und aus meiner Sicht zunächst Aufgabe der Philosophie. Fordert man als Elemente einer Bildung für eine nachhaltige Entwicklung eine transformative Bildung, so wird die Dekonstruktion des naturwissenschaftlichen Denkens auch zu einer Aufgabe für die naturwissenschaftlichen Didaktiken. Ein angemesseneres Ziel für eine an einer nachhaltigen Entwicklung orientierten naturwissenschaftlichen Bildung bestünde aus meiner Sicht darin, SchülerInnen sowohl an den naturwissenschaftlichen Diskurs als auch an einen widerstreitenden Diskurs über Natur heranzuführen. Als Offenhalten eines Widerstreites, der aufgrund der Dominanz des naturwissenschaftlichen Diskurses immer in der Gefahr steht, unartikulierbar zu werden, wäre die Aneignung eines solchen Diskurses selbst als Bildungsprozess im Sinne Kollers zu deuten.

\section{Transformative Bildung im naturwissenschaftlichen Unterricht}

Es stellt sich die Frage, wie sich gegenüber der naturwissenschaftlichen Betrachtung der Natur ein widerstreitender Diskurs bestimmen lässt, der einen alternativen Zugang zur Natur bietet. Eine Möglichkeit dafür bietet der französische Philosoph und Sinologe Jullien (1999, 2004, 2010), der die Grundannahmen des europäischen mit dem chinesischen Denken vergleicht. Ein Vergleich der Grundannahmen europäischen Denkens mit dem chinesischen Denken ist für eine Bestimmung der Eigenheiten naturwissenschaftlichen Denkens vielversprechend: Die neuzeitliche Naturwissenschaft hat sich nur in Europa entwickelt und nicht anderswo. China ist zudem die einzige große Kultur, die, weitestgehend ohne Austausch mit Europa und auch ohne Verwandtschaft der Sprachen, bis zum Beginn des Austausches in der zweiten Hälfte des 16. Jahrhunderts ähnlich entwickelt ist im Bereich der Technik

Kapelari, Suzanne (Hg.), Vierte „Tagung der Fachdidaktik“ 2019:

„Interdisziplinäre fachdidaktische Diskurse zur Bildung für nachhaltige Entwicklung“

(C) 2020 innsbruck university press, ISBN 978-3-99106-019-2, DOI 10.15203/99106-019-2 
(Schiffbau) und gesellschaftlicher Institutionen (Polizei) (Jullien, 2006, S. 7). China ist insofern für Europa die ,fremdeste Kultur“ (Jullien, 2008a, S. 8) und eignet sich damit besonders gut als Kontrastfolie, vor der die unhinterfragten Grundannahmen des europäischen Denkens sichtbar gemacht werden können. Entsprechend bezeichnet Jullien sein eigenes Vorgehen auch als „Dekonstruktion von außen“" (ebd., S. 10). Jullien geht methodisch innerhalb der Sinologie dabei einen Mittelweg. Er geht weder davon aus, dass das chinesische Denken dem europäischen so fremd ist, dass es ohnehin nicht verstanden werden kann, noch davon, dass beide Denkweisen einen gemeinsamen Gegenstand haben (Jullien, 2008b, S. 89f.). Er beschreibt sein methodisches Vorgehen so, dass er im Vergleich der Denktraditionen immer zunächst nach einer beiden Denkweisen gemeinsamen Frage sucht: „Im Rahmen der Frage kann [...] die Spaltung zwischen zwei möglichen Optionen beginnen, die sich nach und nach aus ihr ergeben und allmählich die Alternative bilden, aus der eine Vorstellung der Andersartigkeit hervorgeht" (ebd., S. 94).

Unter den von Jullien herausgearbeiteten Grundannahmen europäischen Denkens lassen sich zwei identifizieren, die in der Naturwissenschaft entfaltet wurden: die Orientierung des Denkens am Modell nach dem Vorbild der euklidischen Geometrie und die Idee, Veränderung in der Natur nach dem Vorbild der Bewegung eines Körpers von einem Anfangspunkt bis zu einem Endpunkt zu betrachten. Beide Grundannahmen wurden unter Rekurs auf die jüngere Wissenschaftsforschung näher untersucht und expliziert (vgl. Kosler, 2016).

Es stellt sich also zunächst die Frage, worin die Orientierung des Denkens am Modell nach dem Vorbild der euklidischen Geometrie besteht. Unter Rekurs auf Netz (1999) konnte gezeigt werden (Kosler, 2016), dass das charakteristische an der euklidischen Geometrie in der Verwendung von Diagrammen besteht. Die Verwendung dieser Diagramme bringt zwei besondere Vorteile mit sich. Zum einen reduziert die Nutzung der Diagramme den Gegenstandsbereich, über den im Zuge der Beweisführung gesprochen wird. Dies geschieht dadurch, dass in den Diagrammen jeweils wenige Punkte durch Buchstaben bezeichnet werden.

Kapelari, Suzanne (Hg.), Vierte „Tagung der Fachdidaktik“ 2019:

„Interdisziplinäre fachdidaktische Diskurse zur Bildung für nachhaltige Entwicklung“

(C) 2020 innsbruck university press, ISBN 978-3-99106-019-2, DOI 10.15203/99106-019-2 
In der Beweisführung wird nur auf eine kleine Anzahl geometrischer Objekte wie Punkte, Linien oder Flächen Bezug genommen. Sie alle können über die ins Diagramm eingezeichneten Buchstaben bezeichnet werden. Zum zweiten steht das jeweilige Diagramm für eine beliebig große Zahl ähnlicher geometrischer Objekte, die mit dem im Diagramm repräsentierten Objekt diejenigen Eigenschaften teilen, die im Rahmen der Beweisführung angenommen werden. Dies ermöglicht es am Ende der Beweisführung der Leserin und dem Leser, sich davon zu überzeugen, dass der am jeweiligen Diagramm geführte Beweis unverändert auch für all diese geometrischen Objekte, für die das Diagramm als Stellvertreterobjekt fungiert, geführt werden könnte, und so der Beweis allgemeingültig ist. Die Verwendung der Diagramme ermöglicht es damit, den Gegenstandsbereich für die Zwecke der Beweisführung zunächst auf sehr wenige Objekte $\mathrm{zu}$ reduzieren und dennoch am Ende allgemeingültige Aussagen $\mathrm{zu}$ erhalten. Jullien zufolge entfaltete sich die Orientierung des Denkens am Modell nach dem Vorbild der Geometrie in der neuzeitlichen Naturwissenschaft, indem Galilei einen Weg fand, Naturwissenschaft nach diesem Vorbild zu betreiben.

Die Idee, Veränderung in der Natur nach dem Vorbild der Bewegung eines Körpers zu betrachten, lokalisiert Jullien bereits in der Physik des Aristoteles. Unter Heranziehung einschlägiger Arbeiten der jüngeren Wissenschaftsphilosophie und -geschichte wurde untersucht, wie Galilei diese beiden Grundannahmen des Denkens aufnahm. Es zeigte sich, dass Galilei gerade den Aristotelischen Begriff der Veränderung am Beispiel der Bewegung eines Körpers weiterentwickeln musste, um die Arbeit mit Diagrammen aus der euklidischen Geometrie in die Naturwissenschaft überführen zu können. Nach Aristoteles lassen sich Veränderungen stets durch die Angabe eines Anfangszustandes, eines Endzustandes und des Objektes, an dem sich die Veränderung vollzieht, charakterisieren (Ackrill, 1985). Entsprechend lässt sich das Herunterfallen eines Körpers durch Angabe des Ortes, an dem der Körper losgelassen wird, des Ortes, an dem der Körper am Boden ankommt, und des Körpers selbst charakterisieren. Galilei weicht nun von diesem Veränderungsverständnis $a b$, indem er den Begriff des Geschwindigkeits- 
momentes einführt. Damit lässt sich das Fallen eines Körpers auch in einem Moment charakterisieren. Es ist damit nicht mehr notwendig, einen Anfangsund einen Endzustand anzugeben. Damit lassen sich nun Veränderungen nicht mehr nur als endliche Bewegungen auffassen, sondern als Bewegungszustände.

Dieser Begriff des Geschwindigkeitsmomentes erlaubt es Galilei, ein euklidisches Diagramm zu zeichnen, um das Fallen eines Körpers zu repräsentieren (Galilei, 2007, S. 158f., vgl. Kosler, 2016, S. 224ff.). Dazu zeichnet Galilei eine senkrechte Linie, die die Zeit repräsentiert, die der Körper fällt. Senkrecht dazu zeichnet Galilei Linien ein, die das Geschwindigkeitsmoment des fallenden Körpers zum jeweiligen Zeitpunkt repräsentieren. Zusätzlich zeichnet Galilei dann noch eine Bewegung mit konstanter Geschwindigkeit ins Diagramm ein und kann so Beziehungen zwischen diesen beiden Bewegungen herstellen und sein zweites Fallgesetz ableiten. Ein Grundproblem bei der Verwendung von Diagrammen in der Naturwissenschaft bestand zunächst darin, dass Naturwissenschaft seit Aristoteles als Analyse der Bewegung von Körpern aufgefasst wurde, sich in den Diagrammen der euklidischen Geometrie jedoch nichts bewegt. Galileis Trick besteht insofern also darin, mit der Zeit und dem Geschwindigkeitsmoment Begriffe heranzuziehen, mit denen sich Bewegung als Aufeinanderfolge von Zuständen beschreiben lässt und dann Diagramme zu finden, deren Elemente einzelne Bewegungszustände repräsentieren.

Naturwissenschaftliches Denken lässt sich nach dieser Analyse als ein Denken charakterisieren, in dem Naturveränderungen nach dem Vorbild der Bewegung von Körpern betrachtet werden und Veränderungsbegriffe verwendet werden, die es erlauben, Veränderungen im Moment und damit auch als Zustand aufzufassen. Damit wird es möglich, endliche Bewegungen als Abfolge von Zuständen aufzufassen und solche Zustände innerhalb einer Bewegung oder zwischen verschiedenen Bewegungen mit Hilfe von Diagrammen zueinander ins Verhältnis $\mathrm{zu}$ setzen und so unbekannte Verhältnisse aus bekannten abzuleiten. Dies ermöglicht es, Naturgesetze aufgrund von plausiblen Annahmen argumentativ herzuleiten. 
Da mit Hilfe von Naturgesetzen Vorhersagen in die Zukunft getroffen werden können und ebensolche Vorhersagen notwendig sind, um Entscheidungen zu treffen, lässt sich nun auch näher beschreiben, in welchem Verhältnis naturwissenschaftliches Denken zur Gestaltung einer nachhaltigen Entwicklung steht. Naturwissenschaftliches Denken ist insofern nützlich für die Gestaltung einer nachhaltigen Entwicklung, als es Vorhersagen in die Zukunft ermöglicht. Und es ist insofern hinderlich für die Gestaltung einer nachhaltigen Entwicklung, als es von einzelnen Objekten und partikularen Zusammenhängen ausgeht und so der Gesamtzusammenhang aller Dinge und Prozesse zunächst ignoriert wird. Diese Analyse ist dabei nicht neu. Neu ist, dass sich mit der zuvor vorgeführten Charakterisierung erklären lässt, warum dies der Fall ist: Es ist die spezifische Verwendung von Diagrammen, die allgemeingültige Argumente und damit die Herleitung von Gesetzen möglich macht und so Aussagen über die Zukunft erlaubt. Und es ist die Verwendung eben dieser Diagramme, die den Gegenstandsbereich des Nachdenkens auf wenige Objekte und Zusammenhänge beschränkt.

Für die Naturwissenschaftsdidaktik stellt sich die Frage, wie es möglich ist, mit SchülerInnen die Vor- und Nachteile naturwissenschaftlichen Denkens auch zu reflektieren. Diese Frage drängt sich angesichts der Abstraktheit meiner Ausführungen zum Wesen naturwissenschaftlichen Denkens besonders auf. Aus meiner Sicht und im Anschluss an Jullien kann das, wie schon die Analyse selbst, in Form einer Kontrastierung passieren. Dazu müsste man Beispiele nach dem Vorbild der Bewegung von Körpern auswählen, SchülerInnen dazu anregen, mit Skizzen zu argumentieren, Veränderungsraten einführen und das eigene Denken immer wieder durch Versuche überprüfen. Dass das Argumentieren mit Skizzen schon in der Primarstufe prinzipiell möglich ist, haben Lehrer und Schauble von der Vanderbilt University in den letzten 20 Jahren immer wieder mit Klassen der Jahrgangsstufen zwei, drei und vier exemplarisch vorgeführt (Lehrer et al. 2000; Lehrer \& Schauble, 2012). Neu zu konzipieren wäre eine Kontrastierung mit einem chinesischen Herangehen für das Nachdenken über Naturveränderungen. Charakteristisch für das traditionelle chinesische Denken ist, dass der Gegenstand des Nachdenkens ganzheitliche 
stille Wandlungsprozesse sind (vgl. Kosler 2016). Das paradigmatische Beispiel dafür sind die Jahreszeiten. Damit sind Veränderungen nicht als Veränderung einer Eigenschaft eines Objektes gefasst, sondern als Veränderung an einer Gesamtheit, die sich an vielen einzelnen Veränderungen manifestiert. Außerdem treten typische Veränderungen zyklisch immer wieder auf. Die Veränderung der Jahreszeiten ist also nicht etwas, das einen definierten Anfangs- und Endzustand hat, sondern etwas, das ohne Anfang und Ende und damit endlos ist. Für die Beschreibung werden zwei miteinander zusammenhängende Faktoren postuliert, die abwechselnd in den Vordergrund und in den Hintergrund treten und sich in den vielen einzelnen Veränderungen manifestieren. Im Falle der Jahreszeiten sind dies die Wärme des Sommers und die Kälte des Winters, die einander abwechseln und die die betrachtete Gesamtheit jeweils auf spezifische Weise umwandeln. Veränderung wird dabei nicht im Sinne einer zeitlichen Ordnung eines Nacheinanders von Zuständen gedacht, sondern ein zeitlicher Aspekt kommt dadurch ins Spiel, dass jeder Moment der Veränderung so verstanden wird, dass es etwas gibt, das geht, und etwas, das kommt. Das Ziel eines solchen Denkens besteht nicht darin, Zusammenhänge zwischen einzelnen Aspekten zu finden und Vorhersagen zu machen, sondern darin, einen solchen Wandlungsprozess wahrzunehmen, die ihn bestimmenden Faktoren zu erfassen und Gelegenheiten aufzuspüren, in denen der Prozess durch geeignete Maßnahmen so beeinflusst werden kann, dass er sich von selbst in eine gewünschte Richtung entwickelt.

\section{Literatur}

Ackrill, J. L. (1985). Aristoteles. Berlin: Gruyter.

Austin, J. L. (1976). How to do Things with Words. Oxford: Oxford University Press.

Bateson, G. (1987). The logical categories of learning and communication. In G. Bateson, Steps to an Ecology of Mind. Collected Essays in Anthropology, Psychiatry, Evolution, and Epistemology (S. 279-308). Northvale: Aronson.

Baumgartner, L. M. (2012). Mezirow's theory of transformative learning from 1975 to present. In E. W. Taylor, P. Cranton and Associates (Ed.), The Handbook of

Kapelari, Suzanne (Hg.), Vierte „Tagung der Fachdidaktik“ 2019:

„Interdisziplinäre fachdidaktische Diskurse zur Bildung für nachhaltige Entwicklung“

(C) 2020 innsbruck university press, ISBN 978-3-99106-019-2, DOI 10.15203/99106-019-2 
Transformative Learning. Theory, Research, and Practice (S. 99-115). San Francisco: Jossey-Bass.

Derrida, J. (1988). Signatur Ereignis Kontext, In J. Derrida, Randgänge der Philosophie (S. 291-314). Wien: Passagen.

Galilei, G. (2007 [1638]). Unterredungen und mathematische Demonstrationen über zwei neue Wissenszweige, die Mechanik und die Fallgesetze betreffend. Ostwalds Klassiker der exakten Wissenschaften, Reprint der Bände 11, 24 und 25, aus dem Italienischen und Lateinischen übers. u. hrsg. v. A. v. Oettingen. Frankfurt a.M.: Deutsch.

Habermas, J. (1968), Erkenntnis und Interesse, In J. Habermas, Technik und Wissenschaft als ,Ideologie“ (S. 146-168). Frankfurt a.M.: Suhrkamp.

Hopkins, C. (2012). Reflections on 20+ Years of ESD, Journal for Education for Sustainable Development 6, 21-34.

Jullien, F. (1999). Über die Wirksamkeit. Berlin: Merve.

Jullien, F. (2004). Über die »Zeit«. Elemente einer Philosophie des Lebens. Zürich: diaphanes.

Jullien, F. (2006). Vortrag vor Managern über Wirksamkeit und Effizienz in China und im Westen. Berlin: Merve.

Jullien, F. (2008a). Umweg über China, In D. Baecker, F. Jullien, P. Jousset, W. Kubin \& P. Pörtner, Kontroverse über China. Sino-Philosophie (S. 7-30). Berlin: Merve.

Jullien, F. (2008b). Eine Dekonstruktion von außen. In D. Baecker, F. Jullien, P. Jousset, W. Kubin \& P. Pörtner. Kontroverse über China. Sino-Philosophie (S. 133159). Berlin: Merve.

Jullien, F. (2010). Die stillen Wandlungen. Berlin: Merve.

Kokemohr, R. (1989). Bildung als Begegnung? Logische und kommunikationstheoretische Aspekte der Bildungstheorie Erich Wenigers und ihre Bedeutung für biographische Bildungsprozesse in der Gegenwart, In O. Hansmann \& W. Marotzki (Hrsg.), Diskurs Bildungstheorie II: Problemgeschichtliche Orientierungen (S. 327-373). Weinheim: Deutscher Studienverlag.

Kokemohr, R. (1992). Zur Bildungsfunktion rhetorischer Figuren. Sprachgebrauch und Verstehen als didaktisches Problem, In Hartmut Entrich \& Lothar Staeck (Hrsg.), Sprache und Verstehen im Biologieunterricht (S. 16-30). Alsbach/Bergstraße: Leuchtturm.

Kokemohr, R. (2007). Bildung als Welt- und Selbstentwurf im Anspruch des Fremden. Eine theoretisch-empirische Annäherung an eine Bildungstheorie, In H.-C. Koller, W. Marotzki \& O. Sanders (Hrsg.), Bildungsprozesse und Fremdheitserfahrung. Beiträge zu einer Theorie transformatorischer Bildungsprozesse (S. 13-68). Bielefeld: Transkript.

Koller, H.-C. (2012). Bildung anders denken. Einführung in die Theorie transformatorischer Bildungsprozesse. Stuttgart: Kohlhammer.

Kapelari, Suzanne (Hg.), Vierte „Tagung der Fachdidaktik“ 2019:

„Interdisziplinäre fachdidaktische Diskurse zur Bildung für nachhaltige Entwicklung“

(C) 2020 innsbruck university press, ISBN 978-3-99106-019-2, DOI 10.15203/99106-019-2 
Kosler, T. (2016). Naturwissenschaftliche Bildung im Elementar- und Primarbereich. Zum naturwissenschaftlichen Denken mit Kindern im Kontext einer nachhaltigen Entwicklung. Bad Heilbrunn: Klinkhardt.

Kosler, T. (2018a). Repräsentieren als naturwissenschaftliche Handlungsweise. Potentiale für den Sachunterricht, In U. Franz, H. Giest, A. Hartinger, A. HeinrichDönges \& B. Reinhoffer (Hrsg.), Handeln im Sachunterricht - konzeptionelle Begründungen und empirische Befunde (S. 85-92). Bad Heilbrunn: Klinkhardt.

Kosler, T. (2018b). Naturwissenschaftliches Denken im Kontrast zu chinesischem Naturdenken, In C. Maurer (Hrsg.), Qualitätsvoller Chemie- und Physikunterricht normative und empirische Dimensionen (S. 332-335). Gesellschaft für Didaktik der Chemie und Physik. Jahrestagung in Regensburg. http://www.gdcp.de/images/tagungsbaende/GDCP Band38.pdf

Kuhn, T. (1976). Die Struktur wissenschaftlicher Revolutionen. Frankfurt a. M.: Suhrkamp.

Kuhn, T. (1981). Was sind wissenschaftliche Revolutionen? München: Carl-Friedrichvon-Siemens-Stiftung.

Lyotard, J.-F. (1986). Das postmoderne Wissen. Ein Bericht. Graz: Böhlau.

Lyotard, J.-F. (1989). Der Widerstreit. München: Fink.

Marotzki, W. (1990). Entwurf einer strukturalen Bildungstheorie. Biographietheoretische Auslegung von Bildungsprozessen in hochkomplexen Gesellschaften. Weinheim: Deutscher Studien Verlag.

Matthews, M. (2004), Thomas Kuhn's impact on science education: What lessons can be learned? Science Education, 88 (1), 90-118.

Mezirow, J. (1978a). Education for Perspective Transformation: Women's Re-entry Programs in Community Colleges. New York: Teachers College, Columbia University.

Mezirow, J. (1978b). Perspective transformation. Adult Education, 28(2), 100-110.

Mezirow, J. (1981). A critial theory of adult learning and education. Adult Education, 32(1), 3-24.

Mezirow, J. (1997). Transformative Erwachsenenbildung. Baltmannsweiler: Schneider.

Netz, R. (1999). The Shaping of Deduction in Greek Mathematics. A Study in Cognitive History. Cambridge: Cambridge University Press.

Österreichische UNESCO-Kommission (2019). Positionspapier zur Umsetzung von SDG 4 in Österreich. https://www.unesco.at/fileadmin/Redaktion/Publikationen/PublikationsDokumente/2019 Positionspapier OEUK Fachbeirat Transformative Bildung.pdf

O'Sullivan, E. (1999). Transformative Learning. Educational Vision for the 21. Century. London: Zed books.

Scott, W., \& Gough, S. (2003). Sustainable Development and Learning. Framing the issues. London: RoutledgeFalmer.

Kapelari, Suzanne (Hg.), Vierte „Tagung der Fachdidaktik“ 2019:

„Interdisziplinäre fachdidaktische Diskurse zur Bildung für nachhaltige Entwicklung“

(C) 2020 innsbruck university press, ISBN 978-3-99106-019-2, DOI 10.15203/99106-019-2 
Searle, J. (1969). Speech Acts: An Essay in the Philosophy of Language. London: Cambridge University Press.

Singer-Brodowski, M. (2016a). Transformative Bildung durch transformatives Lernen. Zur Notwendigkeit der erziehungswissenschaftlichen Fundierung einer neuen Idee. Zeitschrift für internationale Bildungsforschung und Entwicklungspädagogik, 1/2016, 13-17.

Singer-Brodowski, M. (2016b). Transformatives Lernen als neue Theorie-Perspektive in der BNE. In Umweltdachverband GmbH (Hrsg.), Jahrbuch Bildung für nachhaltige Entwicklung - Im Wandel (S. 140-139). Wien: Forum Umweltbildung im Umweltdachverband.

Sterling, S. (2001). Sustainable Education. Re-visioning Learning and Change. Cambridge: Green Books.

Sterling, S. (2003). Whole Systems Thinking as a Basis for Paradigm Change in Ecuation: Explorations in the Eontext of Sustainability. http://www.bath.ac.uk/cree/sterling/sterlingthesis.pdf

Sterling, S. (2008). Sustainable Education - Towards a deep learning response to unsustainability. Policy \& Practice: Education for Sustainable Development, 63-68.

Sterling, S. (2011). Transformative Learning and Sustainability: sketching the conceptual ground. Learning and Teaching in Higher Education, 5, 17-33.

Sterling, S. (2014). Separate Tracks or Real Synergy? Achieving a Closer Relationship between Education and SD, Post-2015. Journal of Education for Sustainable Development, 8(2), 89-112.

Vare, P., \& Scott, W. (2007). Learning for a Change: Exploring the Relationship Between Education and Sustainable Development. Journal of Education for Sustainable Development, 1(2), 191-198.

Wals, A. (2010). Mirroring, Gestaltswitching and transformative social learning. Stepping stones for developing sustainability competence, International Journal of Sustainability in Higher Education, 11(4), 380-390.

Wals, A., \& Dillon, J. (2013). Conventional and Emerging Learning Theories. Implications and Choices for Educational Researchers With a Planetary Consciousness, In R.B. Stevenson, M. Brody, J. Dillon, \& A.E.J. Wals (Eds.), Interantional Handbook of Research on Environmental Education (S. 253-161). New York: Routledge.

Kapelari, Suzanne (Hg.), Vierte „Tagung der Fachdidaktik“ 2019:

„Interdisziplinäre fachdidaktische Diskurse zur Bildung für nachhaltige Entwicklung“

(C) 2020 innsbruck university press, ISBN 978-3-99106-019-2, DOI 10.15203/99106-019-2 